$\therefore$

NASA Technical Memorandum 106845 AIAA-95-0614

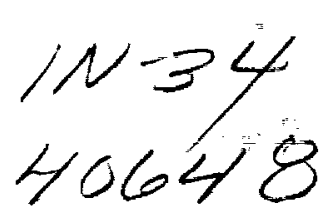

p 12

\title{
Effect of Microgravity on Material Undergoing Melting and Freezing-The TES Experiment
}

David Namkoong, David Jacqmin, and Andrew Szaniszlo

Lewis Research Center

Cleveland, Ohio

Prepared for the

33rd Aerospace Sciences Meeting and Exhibit

sponsored by the American Institute of Aeronautics and Astronautics

Reno, Nevada, January 9-12, 1995

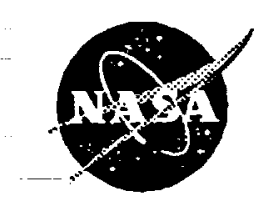

National Aeronautics and Space Administration
(NASA-TM-106845) EFFECT OF MICROGRAVITY ON MATERIAL UNDERGOING

MELTING AND FREEZING: THE TES

EXPERIMENT (NASA. LEWIS RESEarch (enter) $12 \mathrm{p}$
N95-19952

Unclas

$63 / 34 \quad 0040648$ 


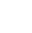


EFFECT OF MICROGRAVITY ON MATERIAL UNDERGOING MELTING AND FREEZING -- THE TES EXPERIMENT

David Namkoong,* David Jacgmin, and Andrew Szaniszlo National Aeronautics and Space Administration

Lewis Research Center

Cleveland, Ohio 44135

\section{Abstract}

This experiment is the first to melt and freeze a high temperature thermal energy storage (TES) material under an extended duration of microgravity. It is one of a series to validate an analytical computer program that predicts void behavior of substances undergoing phase change under microgravity.

Two flight experiments were launched in STS-62. The first, TES-1, containing lithium fluoride in an annular volume, performed flawlessly in the 22 hours of its operation. Results are reported in this paper. A software failure in TES-2 caused its shutdown after 4 seconds.

\section{A computer program, TESSIM, for "Thermal Energy storage Simulation" is being developed to analyze the phenomena occurring within the TES containment vessel. The first order effects, particularly the surface tension forces, have been incorporated into TESSIM.}

*Distinguished Research Associate.
TESSIM validation is based on two types of results. First is the temperature history of various points of the containment structure; and second, upon return from flight, the distribution of the TES material within the containment vessel following the last freeze cycle.

The temperature data over the four cycles showed a repetition of results over the third and fourth cycles. This result is a confirmation that any initial conditions prior to the first cycle had been damped out by the third cycle. The TESSIM simulation showed a close comparison with the flight data.

The solidified TES material distribution within the containment vessel was obtained by a tomography imaging process. The frozen material was concentrated toward the colder end of the annular volume. The TESSIM prediction showed the same pattern.

With the general agreement of TESSIM and the data, a computerized visual representation can be shown which accurately shows the movement and behavior of the void during the entire freezing and melting cycles.

\section{Introduction}

Thermal energy storage (TES), storing heat energy to be used 
at a later time, is a critical feature of constant power, space solar dynamic systems. Utilization of the latent heat of fluoride salts which store and release heat at constant temperature is the preferred choice in systems which employ Brayton or stirling energy conversion subsystems, ref. 1 .

A particular concern in this method of storing heat energy is the change in density of TES materials accompanying the change of phase. Phase change materials (PCM) such as fluoride salts, though suitable for their fusion temperature range, have density differences (solid/liquid) as great as $30 \%$. The salt volume difference in a containment vessel is reflected in the void that grows and shrinks with phase change.

The position of the void can result in either of two extremes when heat impinges upon the containment surface, such as when the space craft emerges from orbital shade to sun. A void adjacent to the heated surface can result in a hot spot. The void remote from the surface, on the other hand, can result in the melting PCM trapped in the still frozen solid. Pressure is exerted on the containment surface by the expanding liquid, deforming it. In this latter case, deformation will occur every orbit, a "ratcheting" effect leading to failure.

Classic designs have avoided these problems by increasing the containment wall thickness. The drawback to this approach is the increased weight for the solar receiver that is already the heaviest component of a solar dynamic system.

The lack of any analysis validated by flight data has prevented engineers from designing lighter weight TES containment structures without sacrificing safety. The TES project is the first to develop a computer program and validate it with flight data.

\section{Experimental Approach}

The TES project was formulated for four experiments with three parameters. These are tabulated in Table 1 .

The parametric selections were based on past and present TES materials and containment designs, but validation of TESSIM would be applicable for any material and geometry where the physical properties are known.

The TES-1 canister material, fig. 1, was Haynes 188 throughout. The TES material, lithium fluoride (LiF), is contained within the annulus.

Heat for melting the TES material was transferred by thermal radiation from a heater sleeve surrounding the canister. The inner surface of the baron nitride sleeve was coated with graphite providing the electrical resistance heating to radiate heat to the canister.

The value of the power input for melting was representative of solar input to a TES canister in a solar receiver. The emitting heat flux was uniform 
except for a narrow axial strip which provided a separate heating intended to influence void behavior.

Cooling to freeze the TES material was accomplished by shutting the power to the heater and by opening the shutter. Heat from the LiF was tranferred to the central rod, to the radiator disc, and to the upper plate of the Getaway Special (GAS) container where the heat was radiated to space.

The heating duration was designed to approximate the orbital period in the sun for a near-Earth orbit -- 60 minutes. The freeze period was determined by the experimental package design. Cooling was approximately 75 minutes.

Initial void placement was determined by the freezing of the LiF before the experimental package was installed in the shuttle. The canister was positioned horizontally so that the initial void was aligned with the strip heater.

The experimental basis for comparison with the analysis was twofold. One was the temperature history of the metal surfaces containing the LiF. Immersing thermocouples in the salt was considered, but because of safety reasons, it was decided that there would be no penetration of the containment wall. Instead, thermocouples were brazed close to the surfaces that were in contact with the TES material.

Thermocouples on the canister (fig. 2) were installed at the quadrant points to read tempera- tures at three cross-sectional planes -- midway and at both ends of the canister.

The second experimental basis for comparison was observation of the frozen LiF upon retrieval of the canister after the flight. The frozen salt was observed non-destructively by a technique of "tomography". similar in concept to a medical "cat-scan", this method allowed observing the solid LiF, and therefore the void pattern, without having to physically section the canister.

\section{Flight Hardware}

Each experimental package has been designed to stand alone, and to operate independently of the shuttle as much as possible. As a complex autonomous payload in a Get-Away-Special (GAS), no power was required from the shuttle and, with flight data stored in the random access memory, no telemetering was needed.

The two experiments on STS-62, TES-1 and TES-2, were mounted along with other experiments on a Hitchhiker-M carrier (fig. 3) which is the OAST-2 primary payload that was placed within the payload bay of the shuttle. The TES experiment occupied 5 cubic feet and weighed 245 pounds prior to placement in the GAS payload container.

The payload consisted of the three hardware subsystems (fig. 4). The top section contained the experiment. The middle section housed the data acquisition and control system (DACS). Also, independent hightemperature control units were 
located in the middle section. They provided added control for maintaining safe maximum temperature level. Finally, the bottom section consisted of a battery box containing 23 silver-zinc cells which provided the electrical energy for the entire package.

The top section or the experiment section was made up of a cylindrical canister assembly, a radiant heater, high-temperature multilayer insulation (MLI), and MLI shutter and drive mechanism as well as temperature measurement instrumentation. Temperature was measured with swaged 20-mil, type $k$ thermocouples. The entire canister assembly was enclosed within the MLI and MLI shutter. The primary components of the canister assembly were the Haynes-188 canister and the thermal radiator disc. Each canister had an annular cross section created by a solid conductor rod along the axis of the canister. The annular cylindrical volume contained the TES salt. The canisters were welded closed in a vacuum after the salt was loaded in the canister.

Heater power levels and MLI shutter opening and closing were controlled by the DACS located in the middle section of the payload. The DACS not only controlled the thermal cycling of the experiment, but also periodically recorded the instrumentation output signals. A central processing unit was used in the DACS to provide the needed data collection speed and processing.
The battery box occupied the lower third section for the experimental payloads. The major part of this section consisted of the battery box itself which contained battery cells. Each cell contained a potassium hydroxide electrolyte. The initial electrical energy level provided by the battery box was about 6300 Wh. The energy in excess of the 3700 Wh expected to be used on orbit, took battery degradation (as a function of time), and possible low temperature at time of launch into account.

\section{operation Sequence}

The TES experiment was activated by an astronaut who began a 5-hour heatup phase. A vacuum environment existed in the GAS payload containers. The heatup phase continued into the initial melting of the TES salt. A total of four melt/freeze cycles were completed over a 10-hour period. Initiation of the experiment
was designed so that the
melt/freeze cycles would coincide with the sleep period of the astronauts. This measure was intended to minimize any acceleration "spikes" during the cycles that may cause movement of the liquid and therefore the void location.

Duration of the heating and cooling cycles were controlled by the DACS reading the thermocouples adjacent to the annulus containing the salt. The heating period was determined when the lowest reading thermocouple 
rose to $5 \mathrm{~K}$ degrees above the melt temperature of the salt. since all other containment temperatures were hotter, all the salt was determined to be molten. At that instant, the heater was turned off and the shutter began opening.

Similarly, duration of cooling was determined when the thermocouple registering the highest temperatures fell to $5 \mathrm{~K}$ degrees below the salt melt temperature. At that point, the signal was sent to the shutter to close, and the power to the heater was turned on.

Flight data were recorded at 5-minute intervals and primarily consisted of temperature and power readings. other data included the time elapsed from the startup of the experiment, time of each data sample, power level, and estimated electrical energy remaining in the battery cells. After the thermal cycles were completed and the experiment section cooled down to approximately $750 \mathrm{~K}, \cdots$ the experiment was deactivated by the astronaut. At this point, a vent valve in the GAS payload container was closed to seal off the GAS container prior to the shuttle de-orbit.

\section{Analysis}

The test temperature data and the location of the frozen TES material were the bases for comparing the predictions of an analytic code. A computer program was developed incorporating all known thermofluid phenomena involved with melting and freezing under microgravity into a simulation of the TES experiment. The code, TESSIM, supersedes a previous program, NORVEX.

TESSIM is a time-accurate three-dimensional code that calculates the behavior of the PCM within the canister. Temperature, enthalpy, the flow field, and cell-averaged mass and volume fraction of solid, liquid, and vapor are all found as functions of time and of location within the canister. The code is finite-volume based. The canister volume is divided into subvolumes for which evolution equations are solved. Mass and enthalpy are used as extensive variables; and temperature, pressure and the phase mass fractions as intensive thermodynamic variables. Fluid velocities are defined at the cell interfaces.

For the Iiquid phase, the code solves the incompressible Navier-stokes equations together with the equation of heat transfer and advection equations for fluid components. Fluid flow is forced by the TES volume change associated with freezing and melting, and by surface tension forces at the vapor/liquid interfaces. The void region is treated as an incompressible fluid with low density and heat capacity. Its volume change as the liquidsolid region expands and shrinks is modeled by including volume sink or source terms in its flow continuity equation.

Describing the interface between void and liquid can be very complex, particularly when subject to abrupt changes in 
topology as the void region evolves. For example, a single void could break up into two voids, or a void could make contact with a wall. Instead of explicitly tracking the interface, the code "senses" the interface by finding regions of large gradients of liquid and void volume fractions. These large gradients are kept large by using slope-steepening convective schemes, ref. 2 . Surface tension is then modeled as a stress that is proportional to these gradients. The magnitude of the surface tension stress can be written as:

$$
|S|=\sigma \frac{|\nabla f(C)|}{f\left(C_{2}\right)-f\left(C_{1}\right)}
$$

where $C$ represents the phase make-up of a control volume and $C_{2}$ and $C_{1}$ are the values of $c$ in phases $\frac{1}{2}$ and $1 . \sigma$ is the surface tension parameter. The surface tension stress acts in the plane of the liquid-void interface. The individual stress tensor components are functions of the interface gradient components times $|S|$. For example, in cartesian coordinates a normal stress $\mathbf{s}_{\mathbf{x x}}$ is given by:

$$
s_{x x}=\left(\left(\frac{\partial f(C)}{\partial y}\right)^{2}+\left(\frac{\partial f(C)}{\partial z}\right)^{2} /|\nabla f(C)|^{2}\right)|S|
$$

and a shear stress $s_{x y}$ by:

$$
s_{x y}=-\left(\frac{\partial f(C)}{\partial x} \frac{\partial f(C)}{\partial y} /|\nabla f(C)|^{2}\right)|S|
$$

Once the surface tension stresses are calculated TESSIM lumps them with the viscous stresses. The total forcing on the flow field is then found by taking the divergence of the total stress field.

The freezing and melting of material is calculated using an enthalpy approach. During a time step the mass in a cell changes through convection and the enthalpy changes through both convection and conduction. The new mass and enthalpy are then fed into the material's thermal equation of state to find the new temperature and the new mass fractions of solid, liquid and vapor. The volume of material in the cell is then found from the volumetric equation of state. If freezing or melting has occurred, the new volume (resulting from the density difference between the liquid and solid states) will not match the volume of the cell. A mismatch is corrected by adding a sink or source term for the volume to the flow continuity equation. correction is not always immediately possible, such as when a melting zone is entirely surrounded by solid. Corrections are delayed if the pressure required to force conformity is unrealistically high or low, or if flow would be required through solid regions.

\section{Results}

The results presented in this paper are preliminary but are indicative of the first order comparisons of the test data and the TESSIM predictions.

Frozen TES Location. The tomographic images shown in fig. 5 show the cross-section of the annulus at seven stations along the axis of the canister. The frozen LiF is concentrated on the radiator end of the canister, the heat sink for the TES material. The shape of the interface, showing a somewhat 
larger void volume at the sector closest to the strip heater, indicates a vestige of the void during the melting period of the cycle.

The predicted formation of the frozen LiF by TESSIM shows a very similar distribution of the solid TES material. The difference appears to be a more lasting influence of the void predicted by TESSIM during freezing.

Temperature History. thermocouples were placed throughout the canister and its surroundings, only the temperatures at the cross-sectional plane midway along the canister axial length is shown. The midpoint diminishes the end effects of the canister and will be a more valid basis of comparison with TESSIM.

Fig. 6 shows the temperatures of the outer containment surface at the quadrant points, for the four melting/freezing cycles. It is clear that repeatability was reached by the fourth cycle. All other temperatures throughout the canister showed the same pattern of repeatability.

A temperature variation during the melt cycle shows an apparent anomaly. As indicated most prominently at the $0^{\circ}$ orientation, the temperature rises, dips, and resumes rising.

\footnotetext{
The TESSIM prediction generally follows the same pattern of rising temperature during melt and dropping temperature during freeze. However, TESSIM does not show the reversal of temperature.
}

Another departure between the data and the analysis is the higher maximum temperature at the end of melting, and lower minimum temperature at the end of freezing predicted by TESSIM.

\section{Discussion of Results}

Development of TESSIM included the capability of visually displaying the analytical behavior of LiF melting and freezing under microgravity. The insights gained from viewing this behavior have provided an appreciation for the complexity of the phenomenon.

The video representation showed a wide-ranging migration of the TES material. The mass alternated between being all liquid at the end of the heating phase filling $95 \%$ of the canister volume and being all solid, concentrated at the cold end of the canister.

Melting and freezing caused significant shifting of the mass during a cycle, and evidently was the reason for the uneven rise of the canister temperature. TESSIM predicted the general profile of temperature, but not the dip during heating.

In general, TESSIM appears to have predicted void location accurately, but not temperature history as well. The TESSIM calculation was performed "blind" =- without any access to the experimental data. It now appears that several oversimplications in implementing details of the TESSIM model caused the temperature history errors. 
one is that radiant heat transfer in the void was less than TESSIM assumed. Higher thermal resistance would have meant higher canister temperature that would have decreased with the migration of liquid PCM across that site.

Another effect was the neglect of radiant heat transfer through the liquid, a significant value.

Most importantly, heat losses through the canister side walls and the bottom surface were underestimated.

Recent calculations made with the corrected boundary conditions and the thermal radiation effects show temperature histories much closer to that of the experiment.

Presently, the chief issue concerning void behavior is the modeling of the wettability of the PCM solid phase. The solid "prefers" to be wet by the liquid phase whereby the PCM liquid phase will advance over a dry solid/liquid interface. This "wicking" effect is not included in current calculations. once included, the void behavior is expected to be more axisymmetric, in agreement with observations.

\section{Conclusions}

The OAST-2 flight has provided the first experimental data of high temperature TES material melting and freezing under an extended duration of microgravity. The test flight data have confirmed, preliminarily, the validity of the analysis.
More detailed analysis of heat transfer within the experimental package, and a further refinement of the computer code, TESSIM, will determine the degree of agreement of the code to the data.

This being the first experiment of high temperature TES melting and freezing under microgravity does not absolutely validate TESSIM, but the comparison of the predictions with data establishes a basic confidence in the code. Other experiments with a combination of a different containment configuration, another TES material, and under a nonwetting condition will determine the extent of the validity of TESSIM.

\section{References}

1. James E. Calogeras, Miles 0 . Dustin and Richard R. secunde "Solar Dynamic Power for Earth Orbital and Lunar Application" NASA TM 104511, Lewis Research Center, cleveland, Ohio. Presented at 26th Intersociety Energy Conversion Conference, Boston, Massachusetts, August 4-9, 1991.

2. H.T. Huynh "Accurate
Upwind Methods for the
Euler Equations" NASA TM
106388, Lewis Research
Center, cleveland, Ohio,
November 1993.


Table 1: Parameters for Melting/Freezing Under Microgravity Flight Experiment

\begin{tabular}{||c|c|c|c||}
\hline Test & $\begin{array}{c}\text { Containment } \\
\text { Geometry }\end{array}$ & Material & Wettedness \\
\hline TES-1 & Annular & LiF & Wetting \\
TES-2 & Annular & LiF/Ca & Wetting \\
TES-3 & Wedge & LiF & Wetting \\
TES-4 & Wedge & LiF & Non-Wetting \\
\hline
\end{tabular}

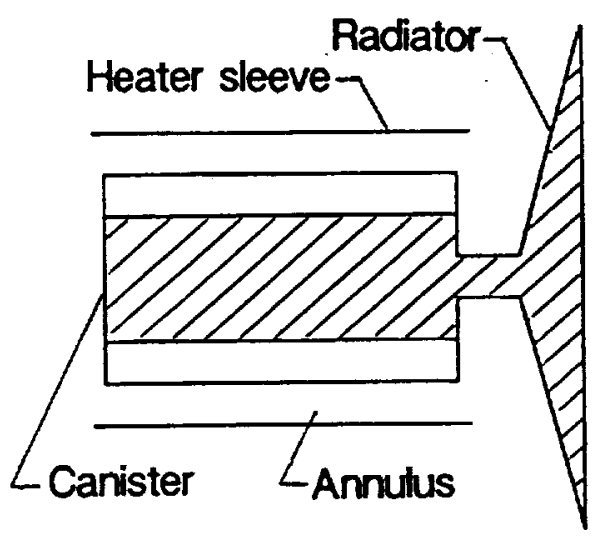

Figure 1: TES-1 Canister Schematic

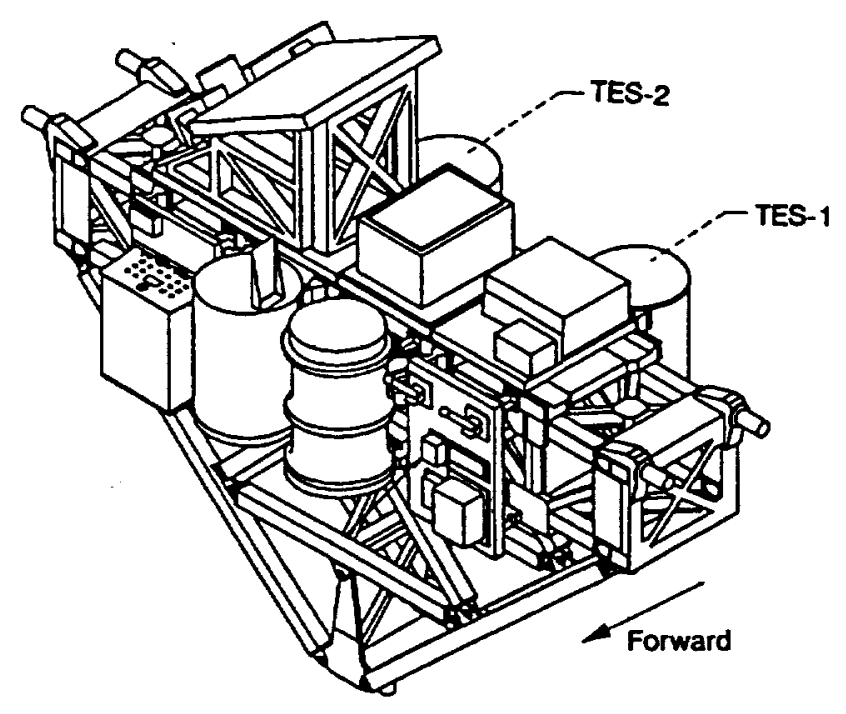

Figure 3: OAST-2 Payload

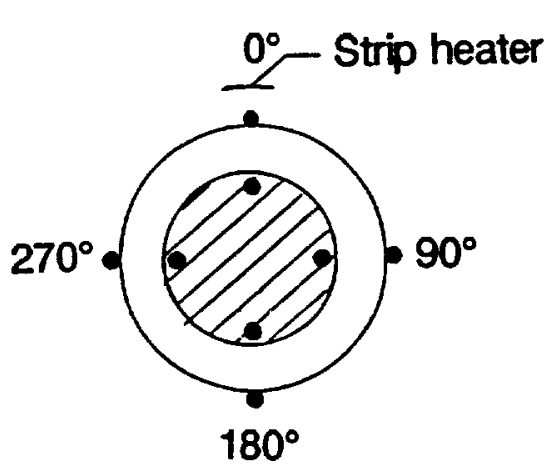

Figure 2: Thermocouple Locations at Three Cross-Sections of Canister as Viewed Toward Radiator

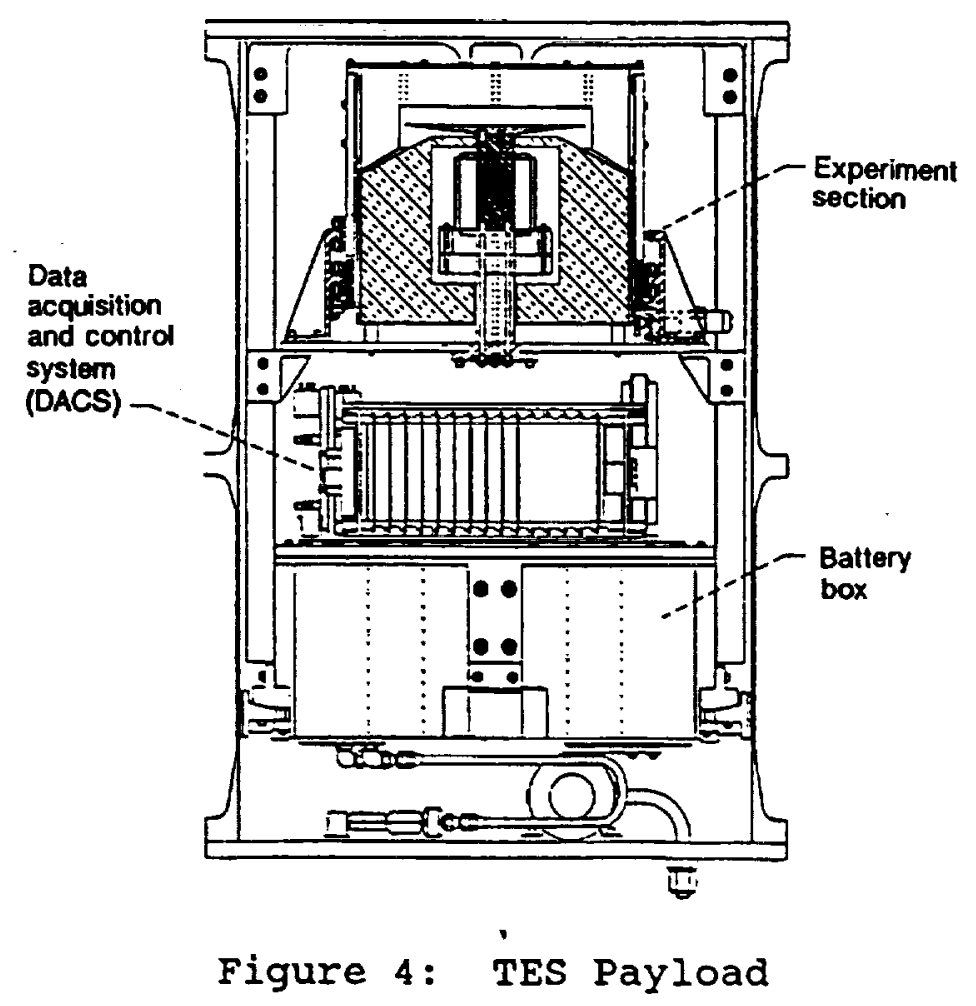




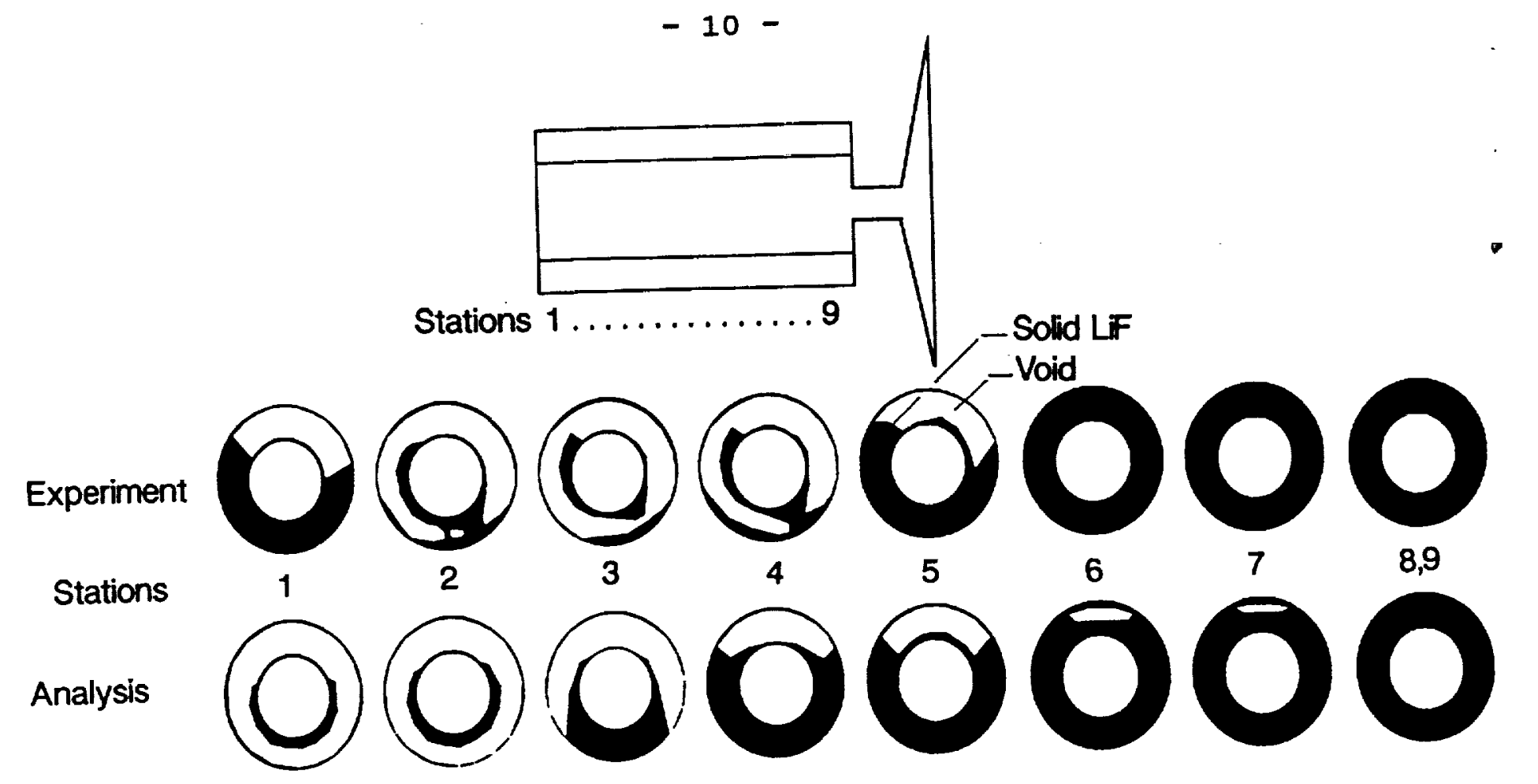

Figure 5: Frozen LiF Distribution in Annular Canister After Four Melt-Freeze Cycles Under Microgravity
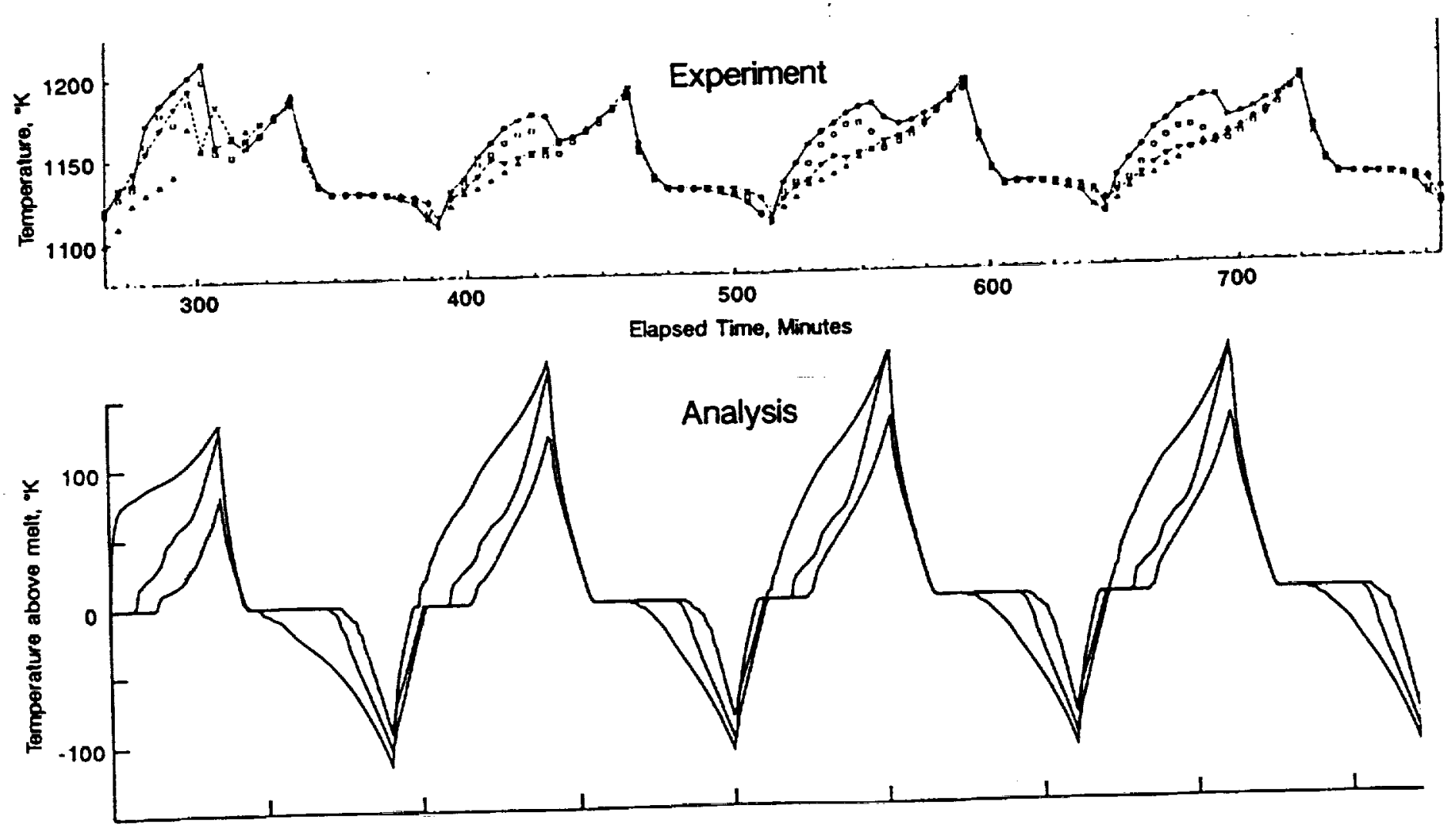

Figure 6: Outer Annular surface Temperatures over Four Melt-Freeze Cycles Under Microgravity. 


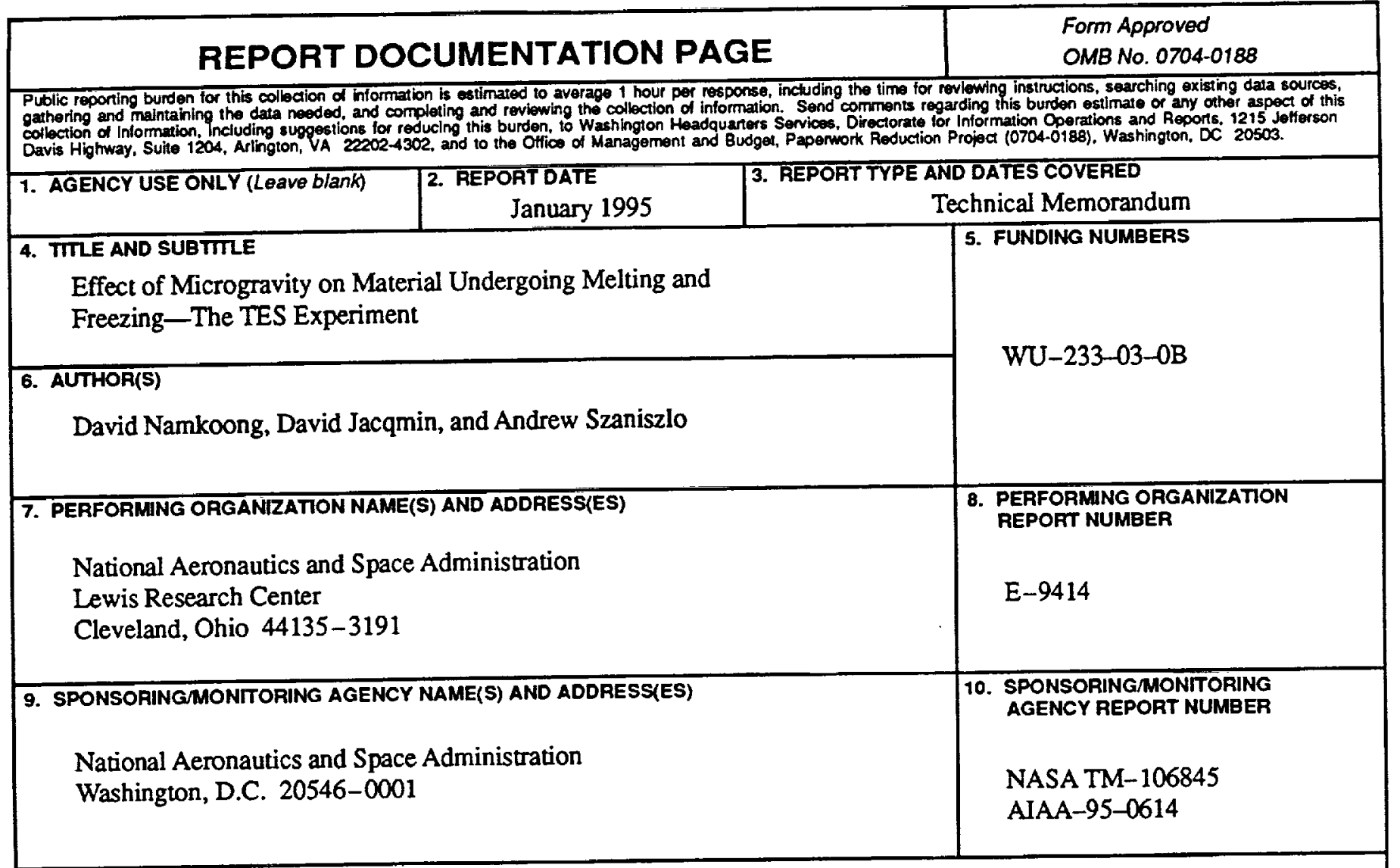

11. SUPPLEMENTARY NOTES

Prepared for the 33rd Aerospace Sciences Meeting and Exhibit sponsored by the American Institute of Aeronautics and Astronautics, Reno, Nevada, January 9-12, 1995. David Namkoong, Distinguished Research Associate; David Jacqmin and Andrew Szaniszlo, NASA Lewis Research Center. Responsible person, David Namkoong, organization code 5490, (216) 433-6301.

\begin{tabular}{|l|l} 
12a. DISTRIBUTIONAVAILABILITY STATEMENT & 12b. DISTRIBUTION CODE
\end{tabular}

Unclassified - Unlimited

Subject Category 34

This publication is available from the NASA Center for Aerospace Information, (301) 621-0390.

13. ABSTRACT (Maximum 200 words)

This experiment is the first to melt and freeze a high temperature thermal energy storage (TES) material under an extended duration of microgravity. It is one of a series to validate an analytical computer program that predicts void behavior of substances undergoing phase change under microgravity. Two flight experiments were launched in STS -62 . The first, TES-1, containing lithium fluoride in an annular volume, performed flawlessly in the 22 hours of its operation. Results are reported in this paper. A software failure in TES-2 caused its shutdown after 4 seconds. A computer program, TESSIM, for "Thermal Energy Storage Simulation" is being developed to analyze the phenomena occurring within the TES containment vessel. The first order effects, particularly the surface tension forces, have been incorporated into TESSIM. TESSIM validation is based on two types of results. First is the temperature history of various points of the containment structure; and second, upon return from flight, the distribution of the TES material within the containment vessel following the last freeze cycle. The temperature data over the four cycles showed a repetition of results over the third and fourth cycles. This result is a confirmation that any initial conditions prior to the first cycle had been damped out by the third cycle. The TESSIM simulation showed a close comparison with the flight data. The solidified TES material distribution within the containment vessel was obtained by a tomography imaging process. The frozen material was concentrated toward the colder end of the annular volume. The TESSIM prediction showed the same pattern. With the general agreement of TESSIM and the data, a computerized visual representation can be shown which accurately shows the movement and behavior of the void during the entire freezing and melting cycles.

14. SUBJECT TERMS

Solar dynamic; Brayton; Thermal Energy Storage (TES); Lithium fluoride; Melting; Freezing

\begin{tabular}{|c|c|}
\hline $\begin{array}{c}\text { 17. SECURTY CLASSIFICATION } \\
\text { OF REPORT } \\
\text { Unclassified }\end{array}$ & $\begin{array}{c}\text { 18. SECURTY CLASSIFICATION } \\
\text { OF THIS PAGE } \\
\text { Unclassified }\end{array}$ \\
\hline
\end{tabular}

NSN 7540-01-280-5500
9. SECURITY CLASSIFICATION OF ABSTRACT Unclassified

15. NUMBER OF PAGES 12

16. PRICE CODE $\mathrm{AO} 3$
20. LIMITATION OF ABSTRACT

Standard Form 298 (Rev. 2-89) Prescrbed by ANSI Sid. 239-18 298.102 